\title{
Improved accuracy in the estimation of blood velocity vectors using matched filtering
}

Jensen, Jørgen Arendt; Gori, P.

\section{Published in:}

Proceedings IEEE Ultrason. Symp.

Link to article, DOI:

10.1109/ULTSYM.2000.921614

Publication date:

2000

Document Version

Publisher's PDF, also known as Version of record

Link back to DTU Orbit

Citation (APA):

Jensen, J. A., \& Gori, P. (2000). Improved accuracy in the estimation of blood velocity vectors using matched filtering. In Proceedings IEEE Ultrason. Symp. (pp. 1525-1530). IEEE.

https://doi.org/10.1109/ULTSYM.2000.921614

\section{General rights}

Copyright and moral rights for the publications made accessible in the public portal are retained by the authors and/or other copyright owners and it is a condition of accessing publications that users recognise and abide by the legal requirements associated with these rights.

- Users may download and print one copy of any publication from the public portal for the purpose of private study or research.

- You may not further distribute the material or use it for any profit-making activity or commercial gain

- You may freely distribute the URL identifying the publication in the public portal

If you believe that this document breaches copyright please contact us providing details, and we will remove access to the work immediately and investigate your claim 


\title{
Improved Accuracy in the Estimation of Blood Velocity Vectors using Matched Filtering
}

\author{
Jørgen Arendt Jensen and Paola Gori \\ Center for Fast Ultrasound Imaging, Department of Information Technology, Build. 344, \\ Technical University of Denmark, DK-2800 Lyngby, Denmark
}

\begin{abstract}
The blood velocity can be estimated by finding the shift in position of the blood scatterers between subsequent ultrasonic pulse emissions through cross-correlation of the received RF signals. Usually only the velocity component along the beam direction is found. It was shown in a previous paper that the complete velocity vector can be found, if the received signals are focused along lines parallel to the direction of the blood flow [1]. A fairly broad beam is emitted in the approach, and this gives rise to a widening in the profiles of the estimated velocity. To reduce this effect, a focused ultrasound emission is used in this study to decrease the size of the beam. The transverse beam lines are then constructed by using filters matched to the response of the individual channels. The filters matched to the points on the focusing lines are obtained by considering the field response of the employed array transducer.

The effect of the processing is studied through simulations with the Field II program. A 64-elements linear array with a center frequency of $3 \mathrm{MHz}$, pitch of $0.3 \mathrm{~mm}$, and element height of $5 \mathrm{~mm}$ is used in the simulations. A parabolic velocity profile is considered for different angles between the flow and the ultrasound beam $(30,45,60$, and 90 degrees $)$. The parabolic flow has a peak velocity of $0.5 \mathrm{~m} / \mathrm{s}$ and the pulse repetition frequency is $3.5 \mathrm{kHz}$. Simulating twenty emissions and calculating the cross-correlation using four pulse-echo lines for each estimate, the parabolic flow profile is found with a standard deviation of $0.014 \mathrm{~m} / \mathrm{s}$ at 45 degrees (corresponding to an accuracy of $2.8 \%$ ) and $0.022 \mathrm{~m} / \mathrm{s}$ (corresponding to an accuracy of $4.4 \%$ ) at 90 degrees, which is transverse to the ultrasound beam. With the approach used in [1], accuracies of $12.6 \%$ and $14.0 \%$, respectively, are obtained in the corresponding situations.
\end{abstract}

\section{Introduction}

Blood velocity estimation in medical ultrasound systems is made by emitting successive ultrasonic pulses and finding the shift in position of the blood scatterers between two consecutive pulses. The shift in position can be determined either by the phase shift between RF signals, or, alternatively, by cross-correlating the received RF signals and estimating the position of the peak in the cross-correlation, which provides the displacement and thereby the velocity [2].

Current commercial systems for blood velocity estimation only find the axial component of the velocity vector, i.e. in the direction perpendicular to the transducer surface. However, many vessels lie approximately parallel to the skin surface, and the main contribution to the velocity vector is the transverse component. Estimators able to provide the entire velocity vector are, thus, needed.

A possible scheme to attain this goal is to use two transducer arrays, or a single large array, in order to beamform in two different directions along which estimates of the velocity components can be found. The velocity vector is then calculated through a triangulation scheme [3]. The problem with this approach is that a small angle between the beams at large depths results in a high variance on the estimate.

Another method is speckle tracking [4] by means of twodimensional cross-correlation, but the method is slow due to the necessity of acquiring two consecutive images. Moreover, averaging is not feasible and erroneous velocities can result as a consequence of false maxima in the correlation function.

A promising approach is the introduction of transverse beam modulation to detect the transverse velocity component, which has been studied in $[5,6,7]$.

In [8], Bonnefous introduced the idea of forming beams parallel to the propagation axis to produce a transverse signal from which the transverse velocity can be found. This approach was extended in [1], where the beamforming is done in reception at a number of points along the direction of the flow vector. These focusing lines are tilted according to the transducer-flow angle, and a velocity distribution can be detected in this way, with each focusing line following scatterers with a unique velocity. A fairly broad beam is emitted in this approach, and this results in a broadening in the profile of the estimated velocity.

In this paper the focusing lines along the direction of the 
flow are not created through delay and sum beamforming, but by applying filters matched to the response of the individual channels, with a different filter for each point in the focusing lines. In this way, the entire transmit-receive process is taken into account, and the problem connected with the use of a broad beam in transmission is overcome.

\section{Matched filter focusing}

Traditional focusing is performed by aligning the responses from the different elements in time, scaling them by an apodization value, and then summing all the responses. This does not take into account that responses from different elements have different waveforms and, thus, does not make an optimized processing of the received signals.

The first step in making an improved focusing is to have a physical understanding of the formation of the received voltage traces. The received voltage trace (RF signal) $v_{r}\left(\vec{r}_{2}, t\right)$ can be written as [9]:

$$
\begin{aligned}
v_{r}\left(\vec{r}_{2}, t\right)= & v_{p e}(t) \star \\
& \int_{V^{\prime}}\left[\frac{\Delta \rho\left(\vec{r}_{1}\right)}{\rho_{0}}-\frac{2 \Delta c\left(\vec{r}_{1}\right)}{c_{0}}\right] h_{p e}\left(\vec{r}_{1}, \vec{r}_{2}, t\right) d^{3} \vec{r}_{1},
\end{aligned}
$$

where $\vec{r}_{2}$ denotes the position of the transducer, $\rho_{0}$ and $c_{0}$ are the mean density and speed of sound of the medium, and $v_{p e}(t)$ is the pulse-echo wavelet, which includes both the transducer excitation and the electro-mechanical impulse response during emission and reception of the pulse. The scattering of the signal originates from the density perturbations $\Delta \rho\left(\vec{r}_{1}\right)$ and speed of sound perturbations $\Delta c\left(\vec{r}_{1}\right)$ in the tissue at the location designated by $\vec{r}_{1}$. The scattering terms are convolved with the spatial pulse-echo impulse response of the transducer designated by $h_{p e}\left(\vec{r}_{1}, \vec{r}_{2}, t\right)$, which describes how the echoes from the individual point scatterers are weighted and filtered by the transducer. The received signal is constructed by a spatial convolution, which sums up the contributions from all the point scatterers, as shown by the integral in the equation.

The measured signal from a point scatterer is, thus, smoothed in time by $v_{p e}(t)$ and in time and space by $h_{p e}\left(\vec{r}_{1}, \vec{r}_{2}, t\right)$, which describes the spatial extent of the point spread function. The pulse-echo spatial impulse response $h_{p e}$ consists of two terms, that describe the impulse response during transmission $h_{t}\left(\vec{r}_{1}, \vec{r}_{2}, t\right)$ and reception $h_{r}\left(\vec{r}_{2}, \vec{r}_{1}, t\right)$, which can be different. This is often the case for a multi-element transducer, where $h_{t}$ consists of a sum of spatial impulse responses for the individual elements that are amplitude scaled (apodized) and delayed to form the emit focus. The combined spatial impulse response is

$$
h_{t}(\vec{r}, t)=\sum_{i=1}^{N_{e}} a_{i} h_{i}\left(\vec{r}_{1}, t-\Delta_{i}\right),
$$
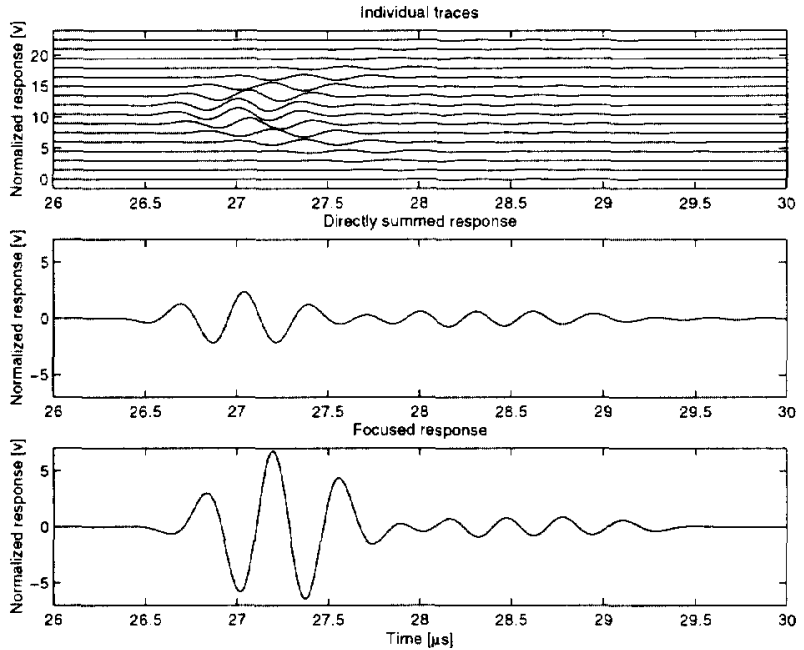

Figure 1: Received signals from the individual elements of a linear array transducer with 16 elements.

where $a_{i}$ denotes the apodization and $\Delta_{i}$ the emit focusing delay. $N_{e}$ is the number of transducer elements. During receive $h_{r}$ is the impulse response for a single element. Equation (2) describes how a single element voltage trace is constructed, and this signal is then passed through the beamformer with the other element signals to dynamically delay and thereby focus the beam in receive. An example of such responses can be seen on Fig. 1. The top graph shows the individual traces from the single elements of an array transducer with $16 \mathrm{el}$ ements, when the transmit focus is at $40 \mathrm{~mm}$ and the point scatterer is placed $20 \mathrm{~mm}$ from the array on its center axis. Both start time and signal shapes vary as a function of element number. A direct summation of the signals is shown in the middle graph and a proper focusing at the point of interest $(20 \mathrm{~mm})$ is shown in the lower figure. The focusing significantly increases the peak amplitude of the response.

From Fig. 2 it can also be seen that a mere alignment of the individual responses is not sufficient to add all parts of the signals in phase. The figure shows the delayed responses received on the center element and on the edge element of the array. The waveform changes as a function of relative position, and the responses are therefore not necessarily in phase, and a delayed response can even subtract instead of add to the focused response. This is a consequence of the spatial and temporal variation of $h_{p e}$.

Proper focusing, thus, needs to address both the distance from the elements to the scattering point through the delay and the actual shape of the response. Ideally each part of the wave should be delayed individually and summed individually to constructively add to the other element signals. This entails filtering each of the element signals with a filter, that is dependent on the position of the element and on the position of the focal point. 


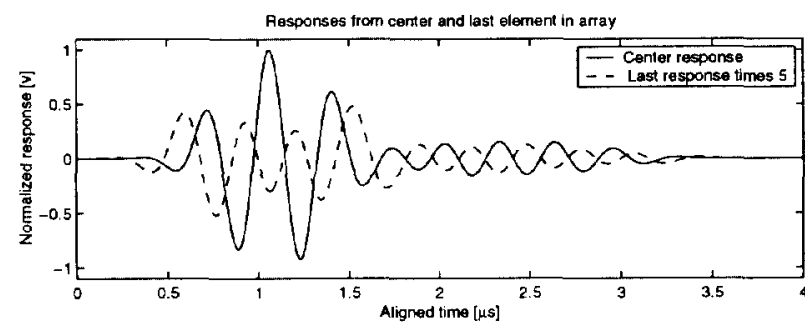

Figure 2: Received signals from the center (solid line) and last element (dashed line) of a linear array transducer with 16 elements, when they are delayed according to their distance to the point scatterer. The last response is multiplied by a factor of 5 .

One option to use on the signals is a matched filter. This filter optimizes the peak signal to noise power, and at the same time aligns the received signals. The time reversal of the impulse response ensures that the resulting signal has a linear phase, and, thus, has the most energy concentrated around its peak value.

\subsection{Spatial matched filter}

The signal from each channel of an array should be processed to align its output with that from the other channels. The received element signals are dependent on the element location and the scatterer's position, and a new matched filter must be used depending on the element and on the scatterer's position. The matched filter $h_{p}\left(\vec{r}_{1}, \vec{r}_{2}, t\right)$ is then given by:

$$
\begin{aligned}
h_{p}\left(\vec{r}_{1}, \vec{r}_{2}, t\right) & =p_{r}\left(\vec{r}_{1}, \vec{r}_{2},-t\right) \\
p_{r}\left(\vec{r}_{1}, \vec{r}_{2}, t\right) & =v_{p e}(t) \star h_{t}\left(\vec{r}_{1}, \vec{r}_{2}, t\right) \star t_{t}\left(\vec{r}_{2}, \vec{r}_{1}, t\right),
\end{aligned}
$$

which is dependent on the transmitter location $\vec{r}_{1}$, the receiver element location $\vec{r}_{2}$, and the electro-mechanical impulse response of the transducer $v_{p e}(t)$. The focusing is then performed by adding the processed signals from all the elements for the different locations in the image as

$$
r_{s}\left(\vec{r}_{i}\right)=\sum_{j=1}^{M} \int_{t_{i j}}^{t_{i j}+\Delta T_{i j}} v_{r}\left(\vec{r}_{j}, t\right) p_{r}\left(\vec{r}_{i}, \vec{r}_{j}, t\right) d t
$$

where $i$ designates the point in the image, $j$ is the element number of the transducer, $t_{i j}$ is the start of the response, and $\Delta T_{i j}$ is the duration of the matched filter. The convolution integral in the equation is replaced by a correlation, since the time reversal of the response is canceled by the time reversal in the convolution.

An example of processed signals can be seen in Fig. 3, where the alignment of the main responses is clearly seen. It can also be seen how responses further away from point scatterer are severely attenuated due to the filters, which shows
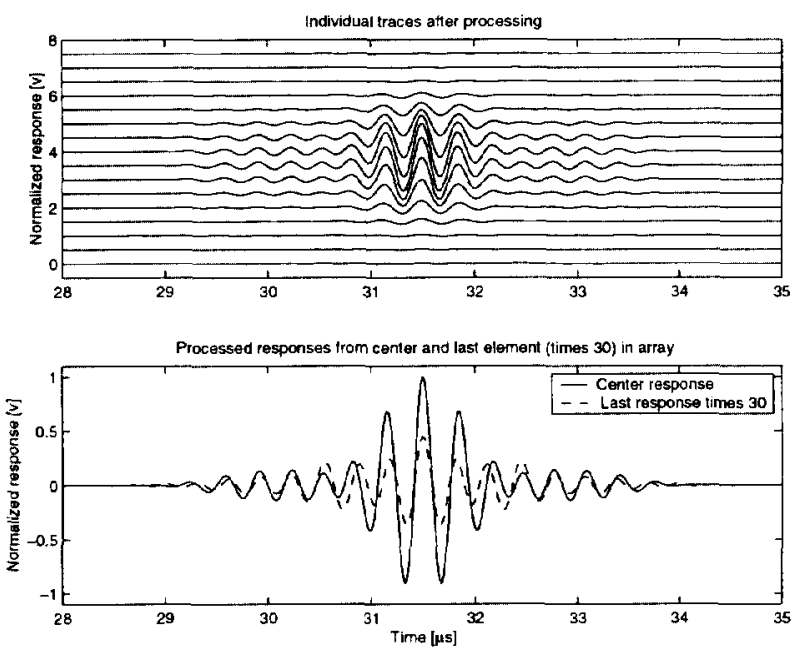

Figure 3: Processed signals from the array transducer with 16 elements. The top graph shows the individual responses and the bottom is from the center element and the last element. The last response is multiplied by a factor of 30 .

how apodization is automatically handled by the approach. All the responses have the characteristics of an autocorrelation function, due to the matched filter, which essentially amounts to a correlation with the assumed response (see Eqn. (4)).

It should be noticed that (4) can be used for any image point, and that it is only necessary to process the points in the image, that must be displayed on the screen. The approach does not put any restrictions on the transducer geometry, excitation, or impulse response, and can be used for both multi-element arrays and single element transducers, as long as the single element is moved compared to the scattering points during the imaging process in $e . g$. a polar scan. It can also be employed in a transverse beam formation for velocity estimation.

The approach improves on the focusing, if the pulse-echo spatial impulse responses are significantly different from a delta function. Normal delay focusing assumes that the geometric impulse response of the transducer is a delta function, and that the alignment can then be done by merely delaying the responses. This is appropriate in the far-field for small element arrays and at the focus for single element transducers. The method will, thus, give the largest improvement in the near field, where long spatial impulse responses are found.

\section{Velocity estimation}

The filtering procedure described in the previous section is applied for points on focusing lines along the direction of the flow. The situation is depicted in Fig. 4, where the lateral beams have to track scatterers with a parabolic velocity 


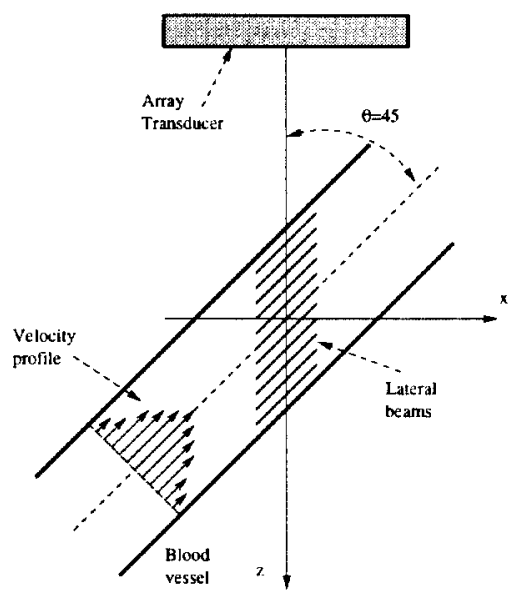

Figure 4: Focusing lines to obtain the lateral focusing lines for a laminar velocity profile.

profile. A signal denoted $R_{s m}[i]$, which is the discrete version of $r_{s m}\left(\vec{r}_{i}\right)$, is obtained for an ultrasonic pulse emitted at time $m T_{\mathrm{prf}}$, where $T_{\mathrm{pr}}$ is the reciprocal of the pulse repetition frequency, and $m$ is an integer indicating the emission number. The distance traveled by a scatterer in the time between two consecutive pulses can then be found through the crosscorrelation of two successive signals $R_{s 1}[i]$ and $R_{s 2}[i]$ :

$$
C_{12}[n]=\frac{1}{L} \sum_{i=1}^{L} R_{s 1}[i] R_{s 2}[i+n]
$$

where $L$ is the number of points in the lateral beam. The signal value $R_{s 2}[i+n]$ is ideally equal to the signal value $R_{s 1}\left[i+n-n_{s}\right]$, where the shift of $n_{s}$ samples accounts for the movement of the scatterers between pulses. It is

$$
n_{s}=\frac{|\vec{v}| T_{\mathrm{prf}}}{\Delta l}
$$

where $\Delta l$ is the distance between two points on the lateral beam. The cross-correlation in (5) is then

$$
C_{12}[n]=\frac{1}{L} \sum_{i=1}^{L} R_{s 1}[i] R_{s 1}\left[i+n-n_{s}\right]=C_{11}\left[n-n_{s}\right]
$$

where $C_{11}[n]$ is the autocorrelation function of $R_{s 1}[i]$. The peak position in the autocorrelation function is an indication of the shift in position of the scatterers, since it occurs at $n=$ $n_{s}$

$$
\max _{n}\left\{C_{11}\left[n-n_{s}\right]\right\}=C_{11}[0]=C_{12}\left[n_{s}\right],
$$

and the estimated shift in position is $\hat{l}_{s}=\Delta l \hat{n}_{s}$. To improve the velocity resolution, $\hat{n}_{s}$ can be substituted by an interpolated value. In the present work, the interpolation was done by fitting a parabola to the cross-correlation function around the maximum [10]. Finally, the estimated velocity is obtained

\begin{tabular}{|c|c|c|c|c|c|}
\hline $\begin{array}{c}f_{s} \\
\mathrm{MHz}\end{array}$ & $\begin{array}{c}f_{\text {prf }} \\
\mathrm{Hz}\end{array}$ & $N_{\text {emissions }}$ & $N_{\text {aver }}$ & $\begin{array}{c}\Delta l \\
\mathrm{~mm}\end{array}$ & $\begin{array}{c}\Delta b \\
\mathrm{~mm}\end{array}$ \\
\hline \hline 100 & 3500 & 20 & 4 & 0.01 & 0.1 \\
\hline
\end{tabular}

Table 1: Simulation parameters.

by dividing the estimated shift in position $\hat{l}_{s}$ by the time between pulses

$$
\hat{v}=\frac{\Delta l \hat{n}_{s}}{T_{\mathrm{prf}}} .
$$

The procedure can be repeated for several emissions, and averaging the cross-correlation (assumed to be nearly constant over a number of emissions) can improve the accuracy and reliability of the estimates.

\section{Results}

The performance of the proposed method has been tested through numerical simulations made with the Field II program [11]. A $3 \mathrm{MHz}$ linear array transducer model consisting of 64 elements with a pitch of $0.3 \mathrm{~mm}$ and height of $5 \mathrm{~mm}$ has been used to simulate the transmit and receive fields. The beam in transmission is not focused in the first example, and a Hanning apodization shades the aperture in transmission. In reception, the processing described in the previous sections is applied. The blood flow has been simulated by shifting a group of scatterers contained in a vessel of radius $5 \mathrm{~mm}$ between successive emissions. The vessel contains 5,400 point scatterers and its center is $30 \mathrm{~mm}$ from the transducer surface. A parabolic velocity profile, with a maximum velocity of $0.5 \mathrm{~m} / \mathrm{s}$ at the center of the vessel, has been simulated. Different angles between the vessel and the beam have been considered $(30,45,60$ and 90 degrees). The pulse repetition frequency $f_{\text {prf }}$ is $3.5 \mathrm{kHz}$. Twenty emissions have been simulated ( $\left.N_{\text {emissions }}\right)$, and the cross-correlation is calculated for four pulse-echo lines $\left(N_{\text {aver }}\right)$. The sampling interval over the lateral beams $\Delta l$ is $0.01 \mathrm{~mm}$ and the distance $\Delta b$ between the lateral beams is $0.1 \mathrm{~mm}$. The parameters used in the simulations are summarized in Table 1.

Table 2 shows the relative mean and standard deviation of the difference between the true profile and the estimated one obtained with the proposed approach and with the one described in [1]. The angle for the flow has been preset to the correct value and is currently not estimated by the approach. Beam-flow angles of 30,45, 60 and 90 degrees have been simulated. An unfocused transmit beam has been used, and it is seen how the new approach in general gives a reduction by a factor 4.5 to 3.2 in the standard deviation.

Examples of velocity estimates for $\theta=45^{\circ}$ and $\theta=90^{\circ}$ are given in Figs. 5 and 6. A slightly worse result is seen 
for focused emission, which has an improved signal to noise ratio because of the focused emission.

Table 3 shows the results, when a focused emitted field is used. A significantly higher energy is then present at the vessel. The table shows the percent deviation from the true profile. It can be seen that the error increases, when the focus is close to the vessel for a purely transverse flow, but that it never exceeds $11 \%$ even at 90 degrees.

It can be seen that the differences of the values in Tables 2 and 3 are very small except for the case of purely transverse flow $\left(\theta=90^{\circ}\right)$. To clarify this point, a curve of the relative standard deviation of the difference between the true profile and the estimated one as a function of the emit focus distance is shown in Fig. 7 for the case of a transverse flow. The graph has been made by estimating the blood velocity over a range of $2 \mathrm{~mm}$ around the center of the vessel. For this reason, the values given for the standard deviation are not directly comparable with those provided in the tables, but they give an idea of the relative influence of the emit focus in the estimation. Since the transition distance from near to far field for the array used in the simulations is at $z=127 \mathrm{~mm}$, the part of the curve that is beyond this value gives information about the performance for an unfocused transmit beam.

\section{Conclusions}

A post-processing approach to focus lateral beams has been presented. The lateral beams track a distribution of scatterers in order to find their velocities. It has been shown that by giving the beam-flow angle as an input, relative accuracies within $4 \%$, when using four pulse emissions, can be achieved for unfocused fields and less than $11 \%$ for focused fields. The new approach makes it possible to use focused fields, and gives an increase in performance by a factor of 3 to 4 compared to the focusing approach used in [1].

\begin{tabular}{|c|c|c|c|c|}
\hline$\theta[\mathrm{deg}]$ & 30 & 45 & 60 & 90 \\
\hline \hline$\frac{E\{\hat{v}-v\}}{\nu_{0}}[\%]$ & 4.8 & 3.6 & 3.8 & 2.8 \\
$\frac{E\{\hat{v}-\nu\}}{\nu_{0}}[1]$ & 24.2 & 15.8 & 15.2 & 10.0 \\
$\frac{\sigma\{\hat{v}-v\}}{\nu_{0}}[\%]$ & 3.4 & 2.8 & 3.4 & 4.4 \\
$\frac{\sigma\{\hat{v}-v\}}{\nu_{0}}[1]$ & 15.2 & 12.6 & 14.4 & 14.0 \\
\hline
\end{tabular}

Table 2: Relative mean and standard deviations of the differences between the true profiles and the estimated ones obtained with the proposed approach and with the one described in [1]. An angle of 90 degrees corresponds to a purely transverse flow.
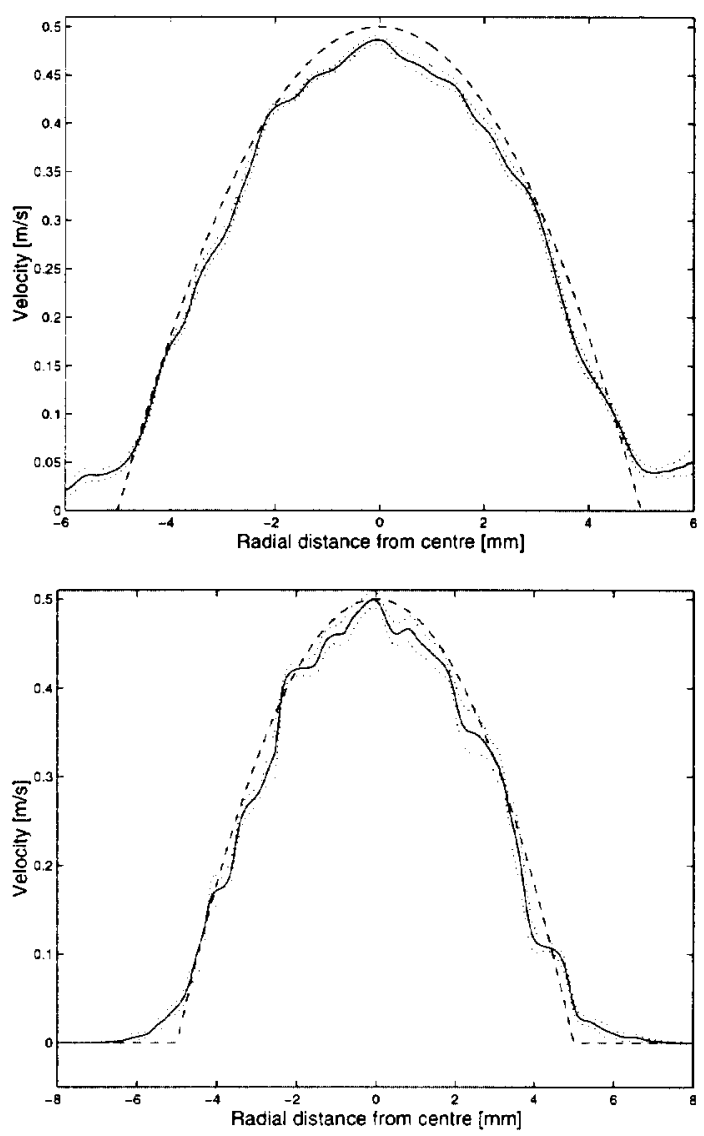

Figure 5: Estimated velocity profiles for $\theta=45^{\circ}$ for unfocused emission (top) and focused emission at $30 \mathrm{~mm}$ (bottom). The dashed line indicates the true velocity profile, the continuous line the mean of the estimates and the dotted lines are the mean \pm one standard deviation.

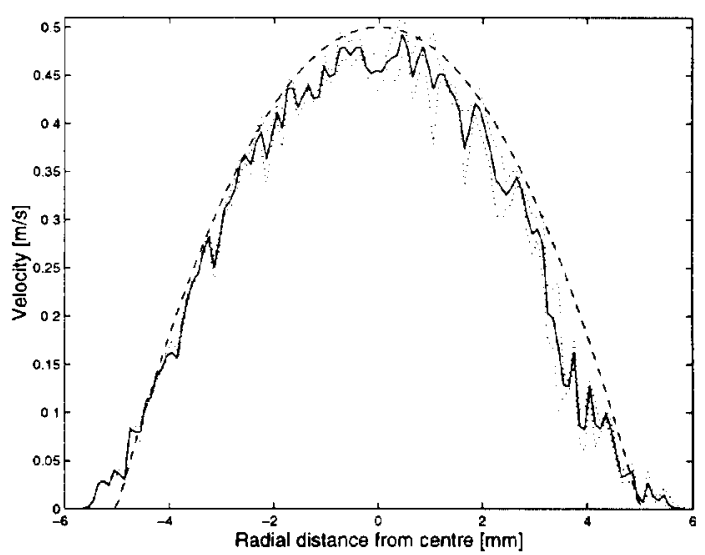

Figure 6: The same as Fig. 5 (top), but for $\theta=90^{\circ}$. 


\section{Acknowledgment}

This work was supported by grant 9700883 and 9700563 from the Danish Science Foundation, by B-K Medical A/S, Gentofte, Denmark and by the Danish Research Academy.

\begin{tabular}{|l|r|r|r|r|r|r|}
\hline $\begin{array}{l}\theta[\mathrm{deg}] \\
\text { Focus [mm] }\end{array}$ & $\begin{array}{r}45 \\
40\end{array}$ & $\begin{array}{r}60 \\
40\end{array}$ & $\begin{array}{r}60 \\
100\end{array}$ & $\begin{array}{r}90 \\
40\end{array}$ & $\begin{array}{r}90 \\
100\end{array}$ & $\begin{array}{r}90 \\
150\end{array}$ \\
\hline \hline$\frac{E\{\hat{v}-v\}}{v_{0}}[\%]$ & -0.63 & -0.53 & -0.82 & -7.3 & -8.7 & -7.0 \\
$\frac{\sigma\{\hat{v}-v\}}{v_{0}}[\%]$ & 2.00 & 2.65 & 1.82 & 10.7 & 8.1 & 9.0 \\
\hline
\end{tabular}

\section{References}

[1] J. A. Jensen and I. R. Lacasa. Estimation of blood velocity vectors using transverse ultrasound beam focusing and cross-correlation. In Proc. IEEE Ultrason. Symp., pages 1493-1497, 1999.

Table 3: Relative mean and standard deviation of the difference between the true profile and the estimated one obtained with a focused emitted field.

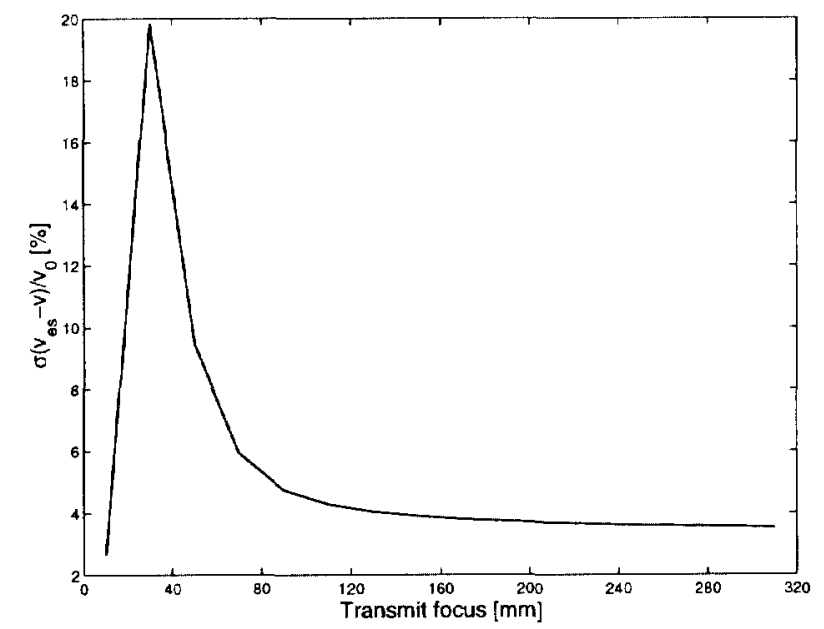

Figure 7: Relative standard deviation of the difference between the true velocity profile and the estimated one, obtained with the proposed approach, as a function of the transmit focus distance.

[2] J. A. Jensen. Estimation of Blood Velocities Using Ultrasound: A Signal Processing Approach. Cambridge University Press, New York, 1996.

[3] M. D. Fox. Multiple crossed-beam ultrasound Doppler velocimetry. IEEE Trans. Son. Ultrason., SU-25:281286,1978 .

[4] G. E. Trahey, J. W. Allison, and O. T. von Ramm. Angle independent ultrasonic detection of blood flow. IEEE Trans. Biomed. Eng., BME-34:965-967, 1987.

[5] P. Munk. Estimation of the 2-D flow vector in ultrasonic imaging: a new approach. Master's thesis, Department of Information Technology, Technical University of Denmark, 1996.

[6] J. A. Jensen and P. Munk. A new method for estimation of velocity vectors. IEEE Trans. Ultrason., Ferroelec., Freq. Contr, 45:837-851, 1998.

[7] M. E. Anderson. Spatial quadrature: a novel technique for multi-dimensional velocity estimation. IEEE Trans. Ultrason., Ferroelec., Freq. Contr., 45:852-681, 1998.

[8] O. Bonnefous. Measurement of the complete (3D) velocity vector of blood flows. In Proc. IEEE Ultrason. Symp., pages 795-799, 1988.

[9] J. A. Jensen. A model for the propagation and scattering of ultrasound in tissue. J. Acoust. Soc. Am., 89:182-191, $1991 \mathrm{a}$.

[10] S. G. Foster. A pulsed ultrasonic flowmeter employing time domain methods. PhD thesis, Dept. Elec. Eng., University of Illinois, Urbana, Ill., 1985.

[11] J. A. Jensen. Field: A program for simulating ultrasound systems. Med. Biol. Eng. Comp., 10th NordicBaltic Conference on Biomedical Imaging, Vol. 4, Supplement 1, Part 1:351-353, 1996b. 